\title{
metal-organic compounds
}

Acta Crystallographica Section E

\section{Structure Reports}

Online

ISSN 1600-5368

\section{$\mu$-Pyrazine-2,5-dicarboxylato-bis- [chlorido $\left(\eta^{6}-p\right.$-cymene)ruthenium(II)] tert-butanol disolvate}

\author{
Noelia M. Sanchez Ballester, Mark R. J. Elsegood* and \\ Martin B. Smith
}

Chemistry Department, Loughborough University, Loughborough, Leicestershire LE11 3TU, England

Correspondence e-mail: m.r.j.elsegood@lboro.ac.uk

Received 12 December 2007; accepted 3 January 2008

Key indicators: single-crystal X-ray study; $T=120 \mathrm{~K}$; mean $\sigma(\mathrm{C}-\mathrm{C})=0.007 \AA$; $R$ factor $=0.049 ; w R$ factor $=0.115 ;$ data-to-parameter ratio $=19.5$.

A new tert-butanol solvate of $\left[\left\{\left({ }^{i} \mathrm{PrC}_{6} \mathrm{H}_{4} \mathrm{Me}\right) \mathrm{RuCl}\right\}_{2}\{\mu-2,5-\right.$ $\left.\left.\operatorname{pyz}(\mathrm{COO})_{2}\right\}\right]$ (pyz = pyrazine $)$ has been crystallized and structurally characterized. The solvate, $\left[\mathrm{Ru}_{2}\left(\mathrm{C}_{10} \mathrm{H}_{14}\right)_{2}\left(\mathrm{C}_{6} \mathrm{H}_{2^{-}}\right.\right.$ $\left.\left.\mathrm{N}_{2} \mathrm{O}_{4}\right) \mathrm{Cl}_{2}\right] \cdot 2 \mathrm{C}_{4} \mathrm{H}_{10} \mathrm{O}$, contains one half-molecule of the ruthenium(II) complex and one molecule of tert-butanol in the asymmetric unit. The complex molecule lies on an inversion centre with the two chlorides trans. In contrast, the previously reported structure was solvent-free. Similar metric parameters are found between the butanol solvate and the solvent-free form and an intermolecular $\mathrm{O}-\mathrm{H} \cdots \mathrm{O}$ hydrogen bond exists between $\quad \mu$-pyrazine-2,5-dicarboxylato-bis[chlorido $\left(\eta^{6}-p\right.$ cymene)ruthenium(II)] and the tert-butanol molecule.

\section{Related literature}

The structure of the solvent-free complex has been reported previously (Govindaswamy et al., 2007). One molecule adopts a trans configuration of the two chloro ligands while the second lies on a twofold axis giving the two chloro ligands a cis configuration. For other related literature, see: Cadierno et al. (2002); Carter et al. (1993); Dann et al. (2006); Dorcier et al. (2005); Drommi et al. (1995); Ganter (2003); Gemel et al. (2000); Grote et al. (2004); Ion et al. (2006); Konar et al. (2004); Lahuerta et al. (1988); Ma et al. (2004); Pinto et al. (2004).

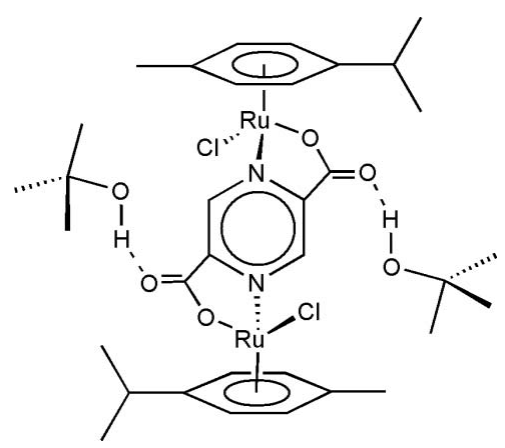

\section{Experimental}

$\begin{array}{ll}\text { Crystal data } & \\ {\left[\mathrm{Ru}_{2}\left(\mathrm{C}_{10} \mathrm{H}_{14}\right)_{2}\left(\mathrm{C}_{6} \mathrm{H}_{2} \mathrm{~N}_{2} \mathrm{O}_{4}\right) \mathrm{Cl}_{2}\right]-} & \beta=91.465(2)^{\circ} \\ \quad 2 \mathrm{C}_{4} \mathrm{H}_{10} \mathrm{O} & V=1833.93(7) \AA^{3} \\ M_{r}=855.80 & Z=2 \\ \text { Monoclinic, } P 2_{1} / c & \text { Mo } K \alpha \text { radiation } \\ a=9.8483(2) \AA & \mu=1.01 \mathrm{~mm}^{-1} \\ b=11.3968(3) \AA & T=120(2) \mathrm{K} \\ c=16.3448(3) \AA & 0.18 \times 0.04 \times 0.03 \mathrm{~mm}\end{array}$

Data collection

Bruker-Nonius Kappa APEXII diffractometer

Absorption correction: multi-scan (SADABS; Sheldrick, 2007)

$T_{\min }=0.839, T_{\max }=0.970$

18492 measured reflections 4195 independent reflections 3594 reflections with $I>2 \sigma(I)$ $R_{\text {int }}=0.046$

Refinement

$R\left[F^{2}>2 \sigma\left(F^{2}\right)\right]=0.049$

$w R\left(F^{2}\right)=0.115$

$S=1.06$

4196 reflections

215 parameters

$\mathrm{H}$-atom parameters constrained

$\Delta \rho_{\max }=2.78{\mathrm{e} \AA^{-3}}^{-3}$

$\Delta \rho_{\min }=-1.04 \mathrm{e}^{-3}$

Table 1

Hydrogen-bond geometry $\left(\AA{ }^{\circ}\right)$.

\begin{tabular}{lllll}
\hline$D-\mathrm{H} \cdots A$ & $D-\mathrm{H}$ & $\mathrm{H} \cdots A$ & $D \cdots A$ & $D-\mathrm{H} \cdots A$ \\
\hline $\mathrm{O} 3-\mathrm{H} 3 \cdots \mathrm{O} 1$ & 0.84 & 1.98 & $2.804(5)$ & 168 \\
\hline
\end{tabular}

Data collection: COLLECT (Hooft, 1998); cell refinement: DENZO (Otwinowski \& Minor, 1997) and COLLECT; data reduction: DENZO and COLLECT; program(s) used to solve structure: SHELXTL (Sheldrick, 2001); program(s) used to refine structure: SHELXTL; molecular graphics: SHELXTL; software used to prepare material for publication: $S H E L X T L$ and local programs.

The authors acknowledge the Loughborough University Development Fund for the provision of a studentship (to NMSB). The authors are also grateful to the EPSRC National Crystallography Service at the University of Southampton for the data collection.

Supplementary data and figures for this paper are available from the IUCr electronic archives (Reference: BV2088). 


\section{References}

Cadierno, V., Díez, J., García-Garrido, S. E., García-Granda, S. \& Gimeno, J. (2002). J. Chem. Soc. Dalton Trans. pp. 1465-1472.

Carter, L., Davies, D. L., Fawcett, J. \& Russell, D. R. (1993). Polyhedron, 12, 1599-1602.

Dann, S. E., Durran, S. E., Elsegood, M. R. J., Smith, M. B., Staniland, P. M., Talib, S. \& Dale, S. H. (2006). J. Organomet. Chem. 691, 4829-4842.

Dorcier, A., Dyson, P. J., Gossens, C., Rothlisberger, U., Scopelliti, R. \& Tavernelli, I. (2005). Organometallics, 24, 2114-2123.

Drommi, D., Arena, C. G., Nicolò, F., Bruno, G. \& Faraone, F. (1995). J. Organomet. Chem. 485, 115-121.

Ganter, C. (2003). Chem. Soc. Rev. 32, 130-138.

Gemel, C., John, R., Slugovc, C., Mereiter, K., Schmid, R. \& Kirchner, K. (2000). J. Chem. Soc. Dalton Trans. pp. 2607-2612.

Govindaswamy, P., Therrien, B., Süss-Fink, G., Štěpnička, P. \& Ludvík, J. (2007). J. Organomet. Chem. 692, 1661-1671.

Grote, Z., Scopelliti, R. \& Severin, K. (2004). J. Am. Chem. Soc. 126, 1695916972.
Hooft, R. W. W. (1998). COLLECT. Nonius BV, Delft, The Netherlands. Ion, L., Morales, D., Pérez, J., Riera, L., Riera, V., Kowenicki, R. A. \& McPartlin, M. (2006). Chem. Commun. pp. 91-93.

Konar, S., Manna, S. C., Zangrando, E. \& Chaudhuri, N. R. (2004). Inorg. Chim. Acta, 357, 1593-1597.

Lahuerta, P., Latorre, J., Sanaú, M., Cotton, F. A. \& Schwotzer, W. (1988). Polyhedron, 7, 1311-1316.

Ma, C., Han, Y. \& Zhang, R. (2004). J. Organomet. Chem. 689, 1675-1683.

Otwinowski, Z. \& Minor, W. (1997). Methods in Enzymology, Vol. 276, Macromolecular Crystallography, Part A, edited by C. W. Carter Jr \& R. M. Sweet, pp. 307-326. New York: Academic Press.

Pinto, P., Marconi, G., Heinemann, F. W. \& Zenneck, U. (2004). Organometallics, 23, 374-380.

Sheldrick, G. M. (2001). SHELXTL. Version 6.10. Bruker AXS Inc., Madison, Wisconsin, USA.

Sheldrick, G. M. (2007). SADABS. Version 2007/2. University of Göttingen, Germany. 


\section{supporting information}

Acta Cryst. (2008). E64, m309-m310 [doi:10.1107/S1600536808000202]

\section{$\mu$-Pyrazine-2,5-dicarboxylato-bis[chlorido( $\eta^{6}-p$-cymene)ruthenium(II)] tert- butanol disolvate}

\section{Noelia M. Sanchez Ballester, Mark R. J. Elsegood and Martin B. Smith}

\section{S1. Comment}

There has been considerable interest in the chemistry of areneruthenium(II) complexes for a variety of purposes. These range from their interesting and varied coordination chemistry (Cadierno et al., 2002; Drommi et al., 1995) including DNA binding studies (Dorcier et al., 2005) to applications in areas including supramolecular chemistry, as highly selective receptors and catalysis (Dann et al., 2006; Ganter, 2003; Grote et al., 2004; Ion et al., 2006). These organometallic ruthenium(II) fragments have also been used in the synthesis of chiral half-sandwich compounds (Ganter, 2003; Pinto et al., 2004). Pyrazine polycarboxylic acids are excellent ligands for metal coordination (Konar et al., 2004; Ma et al., 2004). Complexes of ruthenium(II) with pyrazine carboxylic acids are known and their redox behaviour has been studied by voltammetric methods (Govindaswamy et al., 2007). We report here the molecular structure of a new tert-butanol solvate of the ruthenium(II) complex $\left[\left\{\left(\eta^{6}-p-{ }^{i} \mathrm{PrC}_{6} \mathrm{H}_{4} \mathrm{Me}\right) \mathrm{RuCl}\right\}_{2}\left\{\mu-2,5-\mathrm{pyz}(\mathrm{COO})_{2}\right\}\right] \backslash$. ${ }^{t} \mathrm{BuOH} \mathbf{1}$. The solvent free structure, 2, which contains one molecule with a trans configuration of the two chloro ligands and a second molecule with twofold symmetry that has two chloro ligands disposed in a cis configuration, has recently been reported (Govindaswamy et al., 2007).

The molecular structure of $\mathbf{1}$ is shown in Figure 1 and shows a typical piano-stool geometry at each ruthenium(II) centre with each metal bonded to an $\eta^{6}-p-{ }^{i} \operatorname{PrC}_{6} \mathrm{H}_{4} \mathrm{Me}$ arene [Ru- $\mathrm{C}_{\text {centroid }} 1.6689$ (16) $\AA$ ], a terminal chloride and a dianionic N,O-chelating pyrazine ligand. The $\mathrm{Ru}-\mathrm{Cl}$ bond length in $\mathbf{1}[2.3975$ (11) $\AA]$ is slightly shorter than that in the trans isomer [2.408 (5) $\AA$ ] of $\mathbf{2}$ yet similar to the cis isomer [2.388 (3) $\AA, 2.399$ (3) $\AA$ ]. The $\mathrm{Ru}-\mathrm{O}$ and $\mathrm{Ru}-\mathrm{N}$ bond distances in 1 [2.099 (3) $\AA$ and 2.097 (3) $\AA$ respectively] are similar to those in the cis isomer of 2 [2.083 (10)/2.109 (9) $\AA$ and 2.102 (7)/2.074 (7) $\AA$ respectively] and with those of other related three-legged piano-stool ruthenium(II) complexes (Carter et al., 1993; Gemel et al., 2000; Lahuerta et al., 1988). The $\mathrm{N}(1)-\mathrm{Ru}(1)-\mathrm{O}(2)$ bite angle in 1 [77.29 (12) $\left.{ }^{\circ}\right]$ is broadly as expected for this type of five-membered chelating ligand. The $\eta^{6}-p-{ }^{i} \mathrm{PrC}_{6} \mathrm{H}_{4} \mathrm{Me}$ arene ring is essentially planar with $\mathrm{C}-\mathrm{C}$ bond lengths in the range 1.392 (6)-1.435 (6) $\AA$. The Ru complex is hydrogen-bonded to a ${ }^{t} \mathrm{BuOH}$ molecule through a strong intermolecular $\mathrm{O}-\mathrm{H} \cdots \mathrm{O}$ interaction.

In summary, we have reported the crystal structure of a new tert-butanol solvate form of [ $\left\{\left(\eta^{6}-p\right.\right.$ $\left.\left.\left.{ }^{i} \mathrm{PrC}_{6} \mathrm{H}_{4} \mathrm{Me}\right) \mathrm{RuCl}\right\}_{2}\left\{\mu-2,5-\mathrm{pyz}(\mathrm{COO})_{2}\right\}\right] 1$ that displays very similar bond lengths and angles around the ruthenium(II) coordination sphere to complex 2 recently published (Govindaswamy et al., 2007).

\section{S2. Experimental}

Crystals of compound 1 were obtained unexpectedly from the experimental procedure outlined here. Boronic acid (0.004 $\mathrm{g}, 0.007 \mathrm{mmol})$ in warm ${ }^{t} \mathrm{BuOH}(10 \mathrm{ml})$ was added dropwise to a solution of $\left[\left\{\left(\eta^{6}-p-{ }^{i} \mathrm{PrC}_{6} \mathrm{H}_{4} \mathrm{Me}\right) \mathrm{RuCl}\right\}_{2}\{\mu-2,5-\right.$ pyz $\left.\left.(\mathrm{COO})_{2}\right\}\right](0.023 \mathrm{~g}, 0.0325 \mathrm{mmol})$ in $\mathrm{CH}_{2} \mathrm{Cl}_{2}(10 \mathrm{ml})$ affording an orange-red solution. The solution was stirred at room temperature for $3 \mathrm{~h}$ and the volume was concentrated to 2-3 ml. Suitable X-ray quality crystals of 1 were obtained 
by slow vapour diffusion of diethyl ether into the concentrated $\mathrm{CH}_{2} \mathrm{Cl}_{2} /{ }^{/} \mathrm{BuOH}$ solution.

\section{S3. Refinement}

$\mathrm{H}$ atoms were placed in geometric positions $(\mathrm{C}-\mathrm{H}$ distance $=0.95 \AA$ for aryl $\mathrm{H} ; 0.98 \AA$ for methine, $1.00 \AA$ for methyl $\mathrm{H}$; and $0.84 \AA$ for $\mathrm{O}-\mathrm{H})$ using a riding model. $U_{\text {iso }}$ values were set to $1.2 U_{\text {eq }}(\mathrm{C})\left(1.5 U_{\text {eq }}(\mathrm{C} / \mathrm{O})\right.$ for methyl $\mathrm{H}$ and $\mathrm{OH}$ atoms respectively).

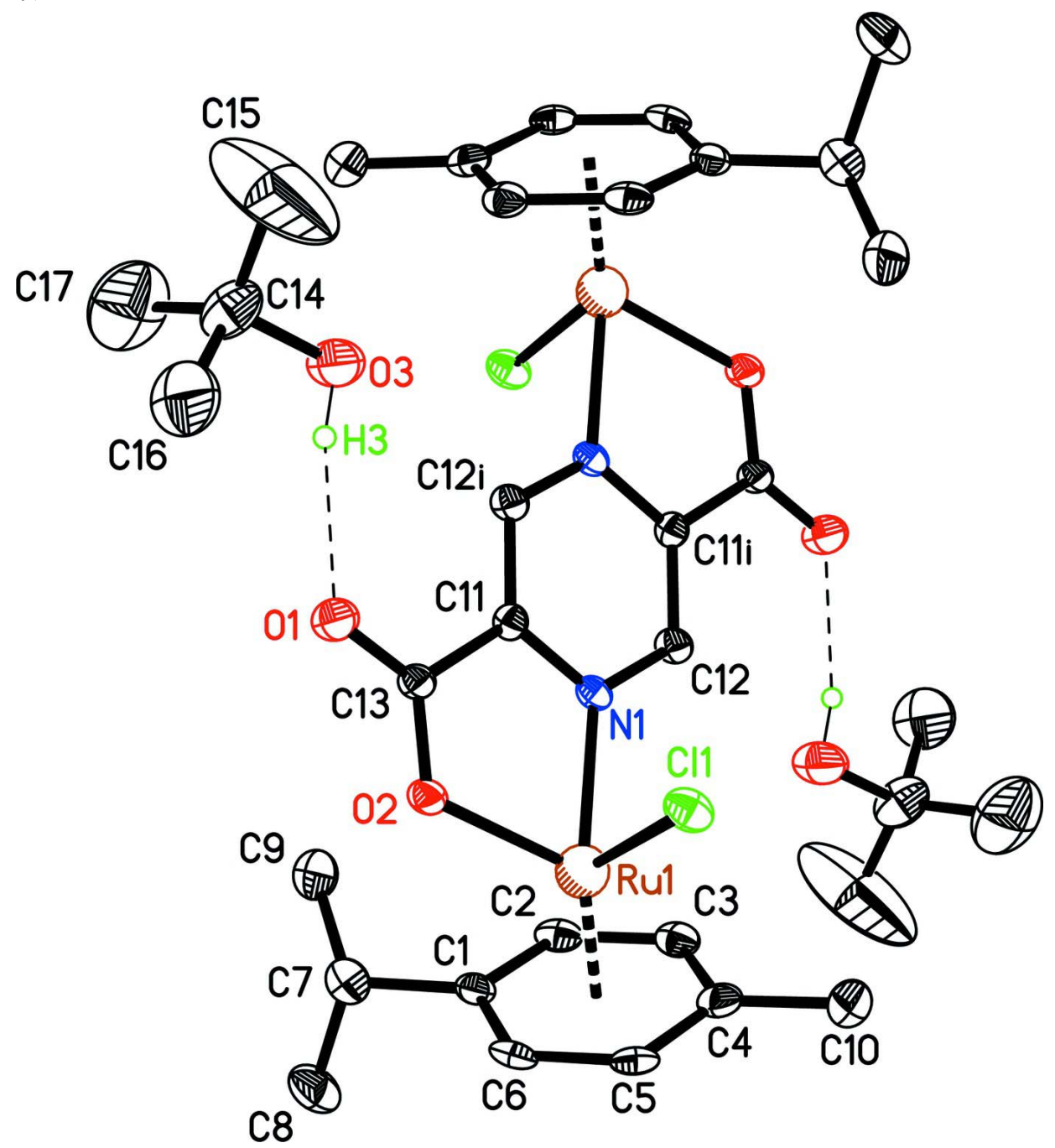

Figure 1

View of 1, showing the atom-labelling scheme. Displacement ellipsoids are drawn at the $50 \%$ probability level. All $\mathrm{H}$ atoms except on $\mathrm{O}(3)$ have been removed for clarity. Symmetry operator: $\mathrm{A}=-x,-y,-z$.

$\mu$-Pyrazine-2,5-dicarboxylato-bis[chlorido( $\eta^{6}$-p-cymene)ruthenium(II)] tert-butanol disolvate

Crystal data

$\left[\mathrm{Ru}_{2}\left(\mathrm{C}_{10} \mathrm{H}_{14}\right)_{2}\left(\mathrm{C}_{6} \mathrm{H}_{2} \mathrm{~N}_{2} \mathrm{O}_{4}\right) \mathrm{Cl}_{2}\right] \cdot 2\left(\mathrm{C}_{4} \mathrm{H}_{10} \mathrm{O}\right)$

$M_{r}=855.80$

Monoclinic, $P 2_{1} / c$

Hall symbol: -P 2ybc

$a=9.8483$ (2) $\AA$

$$
\begin{aligned}
& b=11.3968(3) \AA \\
& c=16.3448(3) \AA \\
& \beta=91.465(2)^{\circ} \\
& V=1833.93(7) \AA^{3} \\
& Z=2
\end{aligned}
$$


$F(000)=876$

$D_{\mathrm{x}}=1.550 \mathrm{Mg} \mathrm{m}^{-3}$

Mo $K \alpha$ radiation, $\lambda=0.71073 \AA$

Cell parameters from 4336 reflections

$\theta=2.9-27.5^{\circ}$

\section{Data collection}

Bruker Nonius APEXII CCD camera on $\kappa$ goniostat diffractometer

Radiation source: Bruker-Nonius FR591 rotating anode

Graphite monochromator

Detector resolution: 4090x4096pixels/62x62mm pixels $\mathrm{mm}^{-1}$ $\varphi$ and $\omega$ scans $\mu=1.01 \mathrm{~mm}^{-1}$

$T=120 \mathrm{~K}$

Rod, brown

$0.18 \times 0.04 \times 0.03 \mathrm{~mm}$

Absorption correction: multi-scan

(SADABS; Sheldrick, 2007)

$T_{\min }=0.839, T_{\max }=0.970$

18492 measured reflections

4195 independent reflections

3594 reflections with $I>2 \sigma(I)$

$R_{\text {int }}=0.046$

$\theta_{\max }=27.5^{\circ}, \theta_{\min }=3.0^{\circ}$

$h=-12 \rightarrow 12$

$k=-14 \rightarrow 14$

$l=-20 \rightarrow 21$

Secondary atom site location: difference Fourier map

Hydrogen site location: inferred from neighbouring sites

$\mathrm{H}$-atom parameters constrained

$w=1 /\left[\sigma^{2}\left(F_{\mathrm{o}}{ }^{2}\right)+(0.0329 P)^{2}+10.5824 P\right]$

where $P=\left(F_{\mathrm{o}}^{2}+2 F_{\mathrm{c}}^{2}\right) / 3$

$(\Delta / \sigma)_{\max }=0.034$

$\Delta \rho_{\max }=2.78{\mathrm{e} \AA^{-3}}^{-3}$

$\Delta \rho_{\min }=-1.04{\mathrm{e} \AA^{-3}}^{-3}$

\section{Special details}

Geometry. All e.s.d.'s (except the e.s.d. in the dihedral angle between two 1.s. planes) are estimated using the full covariance matrix. The cell e.s.d.'s are taken into account individually in the estimation of e.s.d.'s in distances, angles and torsion angles; correlations between e.s.d.'s in cell parameters are only used when they are defined by crystal symmetry. An approximate (isotropic) treatment of cell e.s.d.'s is used for estimating e.s.d.'s involving 1.s. planes.

Refinement. Refinement of $F^{2}$ against ALL reflections. The weighted $R$-factor $w R$ and goodness of fit $S$ are based on $F^{2}$, conventional $R$-factors $R$ are based on $F$, with $F$ set to zero for negative $F^{2}$. The threshold expression of $F^{2}>\sigma\left(F^{2}\right)$ is used only for calculating $R$-factors $(\mathrm{gt})$ etc. and is not relevant to the choice of reflections for refinement. $R$-factors based on $F^{2}$ are statistically about twice as large as those based on $F$, and $R$ - factors based on ALL data will be even larger.

Fractional atomic coordinates and isotropic or equivalent isotropic displacement parameters $\left(\AA^{2}\right)$

\begin{tabular}{lllll}
\hline & $x$ & $y$ & $z$ & $U_{\text {iso }} * / U_{\text {eq }}$ \\
\hline Ru1 & $0.09960(3)$ & $0.28799(3)$ & $0.037062(19)$ & $0.01586(11)$ \\
Cl1 & $0.13823(11)$ & $0.29072(9)$ & $-0.10715(6)$ & $0.0245(2)$ \\
C1 & $0.0607(4)$ & $0.3713(4)$ & $0.1547(2)$ & $0.0199(8)$ \\
C2 & $0.1442(4)$ & $0.2730(4)$ & $0.1682(2)$ & $0.0206(8)$ \\
H2 & 0.1190 & 0.2153 & 0.2069 & $0.025^{*}$ \\
C3 & $0.2668(4)$ & $0.2590(4)$ & $0.1243(3)$ & $0.0231(9)$ \\
H3A & 0.3213 & 0.1912 & 0.1335 & $0.028^{*}$ \\
C4 & $0.3085(4)$ & $0.3447(4)$ & $0.0673(3)$ & $0.0236(9)$ \\
C5 & $0.2248(4)$ & $0.4460(4)$ & $0.0561(3)$ & $0.0217(8)$
\end{tabular}




$\begin{array}{lllll}\text { H5 } & 0.2516 & 0.5056 & 0.0193 & 0.026^{*} \\ \text { C6 } & 0.1045(4) & 0.4591(4) & 0.0982(3) & 0.0216(8) \\ \text { H6 } & 0.0506 & 0.5272 & 0.0893 & 0.026^{*} \\ \text { C7 } & -0.0741(5) & 0.3876(4) & 0.1953(3) & 0.0281(10) \\ \text { H7 } & -0.1365 & 0.4297 & 0.1560 & 0.034^{*} \\ \text { C8 } & -0.0512(6) & 0.4661(5) & 0.2700(3) & 0.0360(11) \\ \text { H8A } & 0.0074 & 0.4256 & 0.3103 & 0.054^{*} \\ \text { H8B } & -0.1387 & 0.4839 & 0.2944 & 0.054^{*} \\ \text { H8C } & -0.0076 & 0.5393 & 0.2533 & 0.054^{*} \\ \text { C9 } & -0.1430(5) & 0.2730(5) & 0.2199(3) & 0.0382(12) \\ \text { H9A } & -0.1494 & 0.2205 & 0.1725 & 0.057^{*} \\ \text { H9B } & -0.2344 & 0.2899 & 0.2392 & 0.057^{*} \\ \text { H9C } & -0.0894 & 0.2352 & 0.2638 & 0.057^{*} \\ \text { C10 } & 0.4356(5) & 0.3291(5) & 0.0202(3) & 0.0363(12) \\ \text { H10A } & 0.5109 & 0.3694 & 0.0487 & 0.054^{*} \\ \text { H10B } & 0.4225 & 0.3623 & -0.0347 & 0.054^{*} \\ \text { H10C } & 0.4565 & 0.2453 & 0.0159 & 0.054^{*} \\ \text { N1 } & 0.0476(3) & 0.1115(3) & 0.01791(19) & 0.0155(6) \\ \text { C11 } & -0.0800(4) & 0.0943(3) & -0.0104(2) & 0.0161(7) \\ \text { C12 } & 0.1276(4) & 0.0178(3) & 0.0281(2) & 0.0177(8) \\ \text { H12 } & 0.2185 & 0.0280 & 0.0478 & 0.021^{*} \\ \text { C13 } & -0.1656(4) & 0.2038(3) & -0.0213(2) & 0.0171(8) \\ \text { O1 } & -0.2818(3) & 0.1959(3) & -0.0502(2) & 0.0293(7) \\ \text { O2 } & -0.1073(3) & 0.2977(2) & 0.00366(18) & 0.0205(6) \\ \text { C14 } & -0.5253(5) & 0.0313(5) & -0.1783(3) & 0.0391(12) \\ \text { C16 } & -0.5410(7) & 0.1587(6) & -0.2005(5) & 0.0596(18) \\ \text { H16A } & -0.4918 & 0.1749 & -0.2506 & 0.089^{*} \\ \text { H16B } & -0.5041 & 0.2073 & -0.1558 & 0.089^{*} \\ \text { H16C } & -0.6375 & 0.1768 & -0.2095 & 0.089^{*} \\ \text { C17 } & -0.6231(8) & 0.0080(8) & -0.1086(5) & 0.081(2) \\ \text { H17A } & -0.7153 & 0.0310 & -0.1260 & 0.122^{*} \\ \text { H17B } & -0.5948 & 0.0538 & -0.0604 & 0.122^{*} \\ \text { H17C } & -0.6218 & -0.0757 & -0.0949 & 0.122^{*} \\ \text { C15 } & -0.5355(14) & -0.0404(12) & -0.2492(7) & 0.176(8) \\ \text { H15A } & -0.4682 & -0.0151 & -0.2887 & 0.264^{*} \\ \text { H15B } & -0.6268 & -0.0333 & -0.2739 & 0.264^{*} \\ \text { H15C } & -0.5184 & -0.1224 & -0.2340 & 0.264^{*} \\ \text { O3 } & -0.3929(4) & 0.0100(3) & -0.1422(3) & 0.0438(10) \\ \text { H3 } & -0.3711 & 0.0663 & -0.1114 & 0.066^{*} \\ & & & & \end{array}$

Atomic displacement parameters $\left(\AA^{2}\right)$

\begin{tabular}{lllllll}
\hline & $U^{11}$ & $U^{22}$ & $U^{33}$ & $U^{12}$ & $U^{13}$ & $U^{23}$ \\
\hline Ru1 & $0.01907(17)$ & $0.01018(16)$ & $0.01823(17)$ & $0.00040(12)$ & $-0.00136(11)$ & $-0.00046(12)$ \\
C11 & $0.0329(5)$ & $0.0211(5)$ & $0.0194(5)$ & $0.0014(4)$ & $0.0014(4)$ & $-0.0001(4)$ \\
C1 & $0.0221(19)$ & $0.0154(19)$ & $0.022(2)$ & $-0.0005(15)$ & $-0.0016(16)$ & $-0.0046(16)$ \\
C2 & $0.028(2)$ & $0.017(2)$ & $0.0160(19)$ & $-0.0009(16)$ & $-0.0094(16)$ & $-0.0006(15)$ \\
C3 & $0.023(2)$ & $0.019(2)$ & $0.027(2)$ & $0.0026(16)$ & $-0.0100(17)$ & $-0.0034(17)$
\end{tabular}




$\begin{array}{lllllll}\text { C4 } & 0.023(2) & 0.023(2) & 0.025(2) & -0.0061(17) & -0.0033(16) & -0.0053(17) \\ \text { C5 } & 0.029(2) & 0.0116(18) & 0.025(2) & -0.0073(16) & -0.0016(17) & -0.0005(16) \\ \text { C6 } & 0.031(2) & 0.0112(19) & 0.022(2) & -0.0005(16) & -0.0028(17) & -0.0057(16) \\ \text { C7 } & 0.030(2) & 0.028(2) & 0.027(2) & 0.0027(19) & 0.0020(18) & -0.0011(19) \\ \text { C8 } & 0.049(3) & 0.031(3) & 0.029(2) & 0.003(2) & 0.010(2) & -0.006(2) \\ \text { C9 } & 0.032(3) & 0.039(3) & 0.044(3) & -0.004(2) & 0.012(2) & -0.005(2) \\ \text { C10 } & 0.025(2) & 0.040(3) & 0.044(3) & -0.005(2) & 0.004(2) & -0.012(2) \\ \text { N1 } & 0.0189(15) & 0.0120(15) & 0.0155(15) & 0.0026(12) & 0.0002(12) & 0.0012(12) \\ \text { C11 } & 0.0156(17) & 0.0163(18) & 0.0163(18) & 0.0013(14) & -0.0009(14) & 0.0015(15) \\ \text { C12 } & 0.0193(18) & 0.0127(18) & 0.021(2) & 0.0029(14) & -0.0025(15) & 0.0024(15) \\ \text { C13 } & 0.0175(18) & 0.0118(18) & 0.022(2) & 0.0012(14) & -0.0015(15) & 0.0016(15) \\ \text { O1 } & 0.0230(15) & 0.0192(16) & 0.045(2) & 0.0034(12) & -0.0097(14) & -0.0013(14) \\ \text { O2 } & 0.0194(14) & 0.0138(14) & 0.0280(16) & 0.0032(11) & -0.0038(11) & -0.0024(12) \\ \text { C14 } & 0.033(3) & 0.045(3) & 0.039(3) & -0.004(2) & -0.014(2) & 0.010(2) \\ \text { C16 } & 0.048(4) & 0.052(4) & 0.077(5) & 0.013(3) & -0.018(3) & 0.006(3) \\ \text { C17 } & 0.073(5) & 0.079(6) & 0.092(6) & -0.014(4) & 0.014(5) & 0.027(5) \\ \text { C15 } & 0.225(14) & 0.180(13) & 0.117(9) & 0.140(12) & -0.123(10) & -0.100(9) \\ \text { O3 } & 0.0337(19) & 0.0284(19) & 0.068(3) & 0.0077(15) & -0.0194(18) & -0.0112(18) \\ & & & & & & \end{array}$

Geometric parameters $\left(\AA,{ }^{\circ}\right)$

\begin{tabular}{llll}
\hline $\mathrm{Ru} 1-\mathrm{N} 1$ & $2.097(3)$ & $\mathrm{C} 9-\mathrm{H} 9 \mathrm{~B}$ & 0.9800 \\
$\mathrm{Ru} 1-\mathrm{O} 2$ & $2.099(3)$ & $\mathrm{C} 9-\mathrm{H} 9 \mathrm{C}$ & 0.9800 \\
$\mathrm{Ru} 1-\mathrm{C} 3$ & $2.176(4)$ & $\mathrm{C} 10-\mathrm{H} 10 \mathrm{~A}$ & 0.9800 \\
$\mathrm{Ru} 1-\mathrm{C} 2$ & $2.184(4)$ & $\mathrm{C} 10-\mathrm{H} 10 \mathrm{~B}$ & 0.9800 \\
$\mathrm{Ru} 1-\mathrm{C} 1$ & $\mathrm{C} 10-\mathrm{H} 10 \mathrm{C}$ & 0.9800 \\
$\mathrm{Ru} 1-\mathrm{C} 6$ & $2.187(4)$ & $\mathrm{N} 1-\mathrm{C} 12$ & $1.335(5)$ \\
$\mathrm{Ru} 1-\mathrm{C} 5$ & $2.191(4)$ & $\mathrm{N} 1-\mathrm{C} 11$ & $1.343(5)$ \\
$\mathrm{Ru} 1-\mathrm{C} 4$ & $2.200(4)$ & $\mathrm{C} 11-\mathrm{C} 12^{\mathrm{i}}$ & $1.389(5)$ \\
$\mathrm{Ru} 1-\mathrm{C} 11$ & $2.201(4)$ & $\mathrm{C} 11-\mathrm{C} 13$ & $1.514(5)$ \\
$\mathrm{C} 1-\mathrm{C} 2$ & $2.3975(11)$ & $\mathrm{C} 12-\mathrm{C} 11^{\mathrm{i}}$ & $1.389(5)$ \\
$\mathrm{C} 1-\mathrm{C} 6$ & $1.404(6)$ & $\mathrm{C} 12-\mathrm{H} 12$ & 0.9500 \\
$\mathrm{C} 1-\mathrm{C} 7$ & $1.435(6)$ & $\mathrm{C} 13-\mathrm{O} 1$ & $1.231(5)$ \\
$\mathrm{C} 2-\mathrm{C} 3$ & $1.511(6)$ & $\mathrm{C} 13-\mathrm{O} 2$ & $1.277(5)$ \\
$\mathrm{C} 2-\mathrm{H} 2$ & $1.429(6)$ & $\mathrm{C} 14-\mathrm{C} 15$ & $1.419(10)$ \\
$\mathrm{C} 3-\mathrm{C} 4$ & 0.9500 & $\mathrm{C} 14-\mathrm{O} 3$ & $1.438(6)$ \\
$\mathrm{C} 3-\mathrm{H} 3 \mathrm{~A}$ & $1.417(6)$ & $\mathrm{C} 14-\mathrm{C} 16$ & $1.503(8)$ \\
$\mathrm{C} 4-\mathrm{C} 5$ & 0.9500 & $\mathrm{C} 14-\mathrm{C} 17$ & $1.534(9)$ \\
$\mathrm{C} 4-\mathrm{C} 10$ & $1.428(6)$ & $\mathrm{C} 16-\mathrm{H} 16 \mathrm{~A}$ & 0.9800 \\
$\mathrm{C} 5-\mathrm{C} 6$ & $1.496(7)$ & $\mathrm{C} 16-\mathrm{H} 16 \mathrm{~B}$ & 0.9800 \\
$\mathrm{C} 5-\mathrm{H} 5$ & $1.392(6)$ & $\mathrm{C} 16-\mathrm{H} 16 \mathrm{C}$ & 0.9800 \\
$\mathrm{C} 6-\mathrm{H} 6$ & 0.9500 & $\mathrm{C} 17-\mathrm{H} 17 \mathrm{~A}$ & 0.9800 \\
$\mathrm{C} 7-\mathrm{C} 8$ & 0.9500 & $\mathrm{C} 17-\mathrm{H} 17 \mathrm{~B}$ & 0.9800 \\
$\mathrm{C} 7-\mathrm{C} 9$ & $1.525(6)$ & $\mathrm{C} 17-\mathrm{H} 17 \mathrm{C}$ & 0.9800 \\
$\mathrm{C} 7-\mathrm{H} 7$ & $1.530(7)$ & $\mathrm{C} 15-\mathrm{H} 15 \mathrm{~A}$ & 0.9800 \\
$\mathrm{C} 8-\mathrm{H} 8 \mathrm{~A}$ & 1.0000 & $\mathrm{C} 15-\mathrm{H} 15 \mathrm{~B} 15 \mathrm{C}$ & 0.9800 \\
$\mathrm{C} 8-\mathrm{H} 8 \mathrm{~B}$ & 0.9800 & & 0.9800 \\
$\mathrm{C} 8-\mathrm{H} 8 \mathrm{C}$ & 0.9800 & & 0.8400 \\
& 0.9800 & &
\end{tabular}




\begin{tabular}{|c|c|c|c|}
\hline C9- $\mathrm{H} 9 \mathrm{~A}$ & 0.9800 & & \\
\hline $\mathrm{N} 1-\mathrm{Ru} 1-\mathrm{O} 2$ & $77.29(12)$ & $\mathrm{C} 5-\mathrm{C} 6-\mathrm{Ru} 1$ & $71.9(2)$ \\
\hline $\mathrm{N} 1-\mathrm{Ru} 1-\mathrm{C} 3$ & $97.48(14)$ & $\mathrm{C} 1-\mathrm{C} 6-\mathrm{Ru} 1$ & $70.7(2)$ \\
\hline $\mathrm{O} 2-\mathrm{Ru} 1-\mathrm{C} 3$ & $153.12(15)$ & $\mathrm{C} 5-\mathrm{C} 6-\mathrm{H} 6$ & 119.5 \\
\hline $\mathrm{N} 1-\mathrm{Ru} 1-\mathrm{C} 2$ & $96.53(14)$ & $\mathrm{C} 1-\mathrm{C} 6-\mathrm{H} 6$ & 119.5 \\
\hline $\mathrm{O} 2-\mathrm{Ru} 1-\mathrm{C} 2$ & $115.40(14)$ & $\mathrm{Ru} 1-\mathrm{C} 6-\mathrm{H} 6$ & 130.7 \\
\hline $\mathrm{C} 3-\mathrm{Ru} 1-\mathrm{C} 2$ & $38.27(17)$ & $\mathrm{C} 1-\mathrm{C} 7-\mathrm{C} 8$ & $108.1(4)$ \\
\hline $\mathrm{N} 1-\mathrm{Ru} 1-\mathrm{C} 1$ & $120.00(14)$ & $\mathrm{C} 1-\mathrm{C} 7-\mathrm{C} 9$ & $114.3(4)$ \\
\hline $\mathrm{O} 2-\mathrm{Ru} 1-\mathrm{C} 1$ & $90.86(13)$ & $\mathrm{C} 8-\mathrm{C} 7-\mathrm{C} 9$ & $110.4(4)$ \\
\hline $\mathrm{C} 3-\mathrm{Ru} 1-\mathrm{C} 1$ & $68.67(16)$ & $\mathrm{C} 1-\mathrm{C} 7-\mathrm{H} 7$ & 108.0 \\
\hline $\mathrm{C} 2-\mathrm{Ru} 1-\mathrm{C} 1$ & $37.47(15)$ & $\mathrm{C} 8-\mathrm{C} 7-\mathrm{H} 7$ & 108.0 \\
\hline $\mathrm{N} 1-\mathrm{Ru} 1-\mathrm{C} 6$ & $157.55(15)$ & $\mathrm{C} 9-\mathrm{C} 7-\mathrm{H} 7$ & 108.0 \\
\hline $\mathrm{O} 2-\mathrm{Ru} 1-\mathrm{C} 6$ & $94.69(14)$ & $\mathrm{C} 7-\mathrm{C} 8-\mathrm{H} 8 \mathrm{~A}$ & 109.5 \\
\hline $\mathrm{C} 3-\mathrm{Ru} 1-\mathrm{C} 6$ & $80.13(16)$ & $\mathrm{C} 7-\mathrm{C} 8-\mathrm{H} 8 \mathrm{~B}$ & 109.5 \\
\hline $\mathrm{C} 2-\mathrm{Ru} 1-\mathrm{C} 6$ & $67.69(15)$ & $\mathrm{H} 8 \mathrm{~A}-\mathrm{C} 8-\mathrm{H} 8 \mathrm{~B}$ & 109.5 \\
\hline $\mathrm{C} 1-\mathrm{Ru} 1-\mathrm{C} 6$ & $38.28(15)$ & $\mathrm{C} 7-\mathrm{C} 8-\mathrm{H} 8 \mathrm{C}$ & 109.5 \\
\hline $\mathrm{N} 1-\mathrm{Ru} 1-\mathrm{C} 5$ & $160.06(15)$ & $\mathrm{H} 8 \mathrm{~A}-\mathrm{C} 8-\mathrm{H} 8 \mathrm{C}$ & 109.5 \\
\hline $\mathrm{O} 2-\mathrm{Ru} 1-\mathrm{C} 5$ & $122.04(14)$ & $\mathrm{H} 8 \mathrm{~B}-\mathrm{C} 8-\mathrm{H} 8 \mathrm{C}$ & 109.5 \\
\hline $\mathrm{C} 3-\mathrm{Ru} 1-\mathrm{C} 5$ & $67.62(16)$ & $\mathrm{C} 7-\mathrm{C} 9-\mathrm{H} 9 \mathrm{~A}$ & 109.5 \\
\hline $\mathrm{C} 2-\mathrm{Ru} 1-\mathrm{C} 5$ & $80.04(16)$ & $\mathrm{C} 7-\mathrm{C} 9-\mathrm{H} 9 \mathrm{~B}$ & 109.5 \\
\hline $\mathrm{C} 1-\mathrm{Ru} 1-\mathrm{C} 5$ & $68.30(16)$ & $\mathrm{H} 9 \mathrm{~A}-\mathrm{C} 9-\mathrm{H} 9 \mathrm{~B}$ & 109.5 \\
\hline $\mathrm{C} 6-\mathrm{Ru} 1-\mathrm{C} 5$ & $36.97(16)$ & $\mathrm{C} 7-\mathrm{C} 9-\mathrm{H} 9 \mathrm{C}$ & 109.5 \\
\hline $\mathrm{N} 1-\mathrm{Ru} 1-\mathrm{C} 4$ & $122.58(15)$ & $\mathrm{H} 9 \mathrm{~A}-\mathrm{C} 9-\mathrm{H} 9 \mathrm{C}$ & 109.5 \\
\hline $\mathrm{O} 2-\mathrm{Ru} 1-\mathrm{C} 4$ & $159.86(14)$ & $\mathrm{H} 9 \mathrm{~B}-\mathrm{C} 9-\mathrm{H} 9 \mathrm{C}$ & 109.5 \\
\hline $\mathrm{C} 3-\mathrm{Ru} 1-\mathrm{C} 4$ & $37.78(17)$ & $\mathrm{C} 4-\mathrm{C} 10-\mathrm{H} 10 \mathrm{~A}$ & 109.5 \\
\hline $\mathrm{C} 2-\mathrm{Ru} 1-\mathrm{C} 4$ & $68.83(16)$ & $\mathrm{C} 4-\mathrm{C} 10-\mathrm{H} 10 \mathrm{~B}$ & 109.5 \\
\hline $\mathrm{C} 1-\mathrm{Ru} 1-\mathrm{C} 4$ & $81.87(16)$ & $\mathrm{H} 10 \mathrm{~A}-\mathrm{C} 10-\mathrm{H} 10 \mathrm{~B}$ & 109.5 \\
\hline $\mathrm{C} 6-\mathrm{Ru} 1-\mathrm{C} 4$ & $68.07(16)$ & $\mathrm{C} 4-\mathrm{C} 10-\mathrm{H} 10 \mathrm{C}$ & 109.5 \\
\hline $\mathrm{C} 5-\mathrm{Ru} 1-\mathrm{C} 4$ & $37.87(16)$ & $\mathrm{H} 10 \mathrm{~A}-\mathrm{C} 10-\mathrm{H} 10 \mathrm{C}$ & 109.5 \\
\hline $\mathrm{N} 1-\mathrm{Ru} 1-\mathrm{Cl1}$ & $84.84(9)$ & $\mathrm{H} 10 \mathrm{~B}-\mathrm{C} 10-\mathrm{H} 10 \mathrm{C}$ & 109.5 \\
\hline $\mathrm{O} 2-\mathrm{Ru} 1-\mathrm{Cl1}$ & $85.47(9)$ & $\mathrm{C} 12-\mathrm{N} 1-\mathrm{C} 11$ & $118.1(3)$ \\
\hline $\mathrm{C} 3-\mathrm{Ru} 1-\mathrm{C} 11$ & $120.66(13)$ & $\mathrm{C} 12-\mathrm{N} 1-\mathrm{Ru} 1$ & $127.4(3)$ \\
\hline $\mathrm{C} 2-\mathrm{Ru} 1-\mathrm{C} 11$ & $158.93(12)$ & $\mathrm{C} 11-\mathrm{N} 1-\mathrm{Ru} 1$ & $114.5(3)$ \\
\hline $\mathrm{C} 1-\mathrm{Ru} 1-\mathrm{C} 11$ & $153.45(11)$ & $\mathrm{N} 1-\mathrm{C} 11-\mathrm{C} 12^{\mathrm{i}}$ & $121.0(4)$ \\
\hline $\mathrm{C} 6-\mathrm{Ru} 1-\mathrm{Cl1}$ & $115.75(12)$ & $\mathrm{N} 1-\mathrm{C} 11-\mathrm{C} 13$ & $115.7(3)$ \\
\hline $\mathrm{C} 5-\mathrm{Ru} 1-\mathrm{Cl1}$ & $91.50(12)$ & $\mathrm{C} 12^{\mathrm{i}}-\mathrm{C} 11-\mathrm{C} 13$ & $123.3(3)$ \\
\hline $\mathrm{C} 4-\mathrm{Ru} 1-\mathrm{C} 11$ & $92.66(12)$ & $\mathrm{N} 1-\mathrm{C} 12-\mathrm{C} 11^{\mathrm{i}}$ & $121.0(4)$ \\
\hline $\mathrm{C} 2-\mathrm{C} 1-\mathrm{C} 6$ & $118.2(4)$ & $\mathrm{N} 1-\mathrm{C} 12-\mathrm{H} 12$ & 119.5 \\
\hline $\mathrm{C} 2-\mathrm{C} 1-\mathrm{C} 7$ & $123.1(4)$ & $\mathrm{C} 11^{\mathrm{i}}-\mathrm{C} 12-\mathrm{H} 12$ & 119.5 \\
\hline $\mathrm{C} 6-\mathrm{C} 1-\mathrm{C} 7$ & $118.6(4)$ & $\mathrm{O} 1-\mathrm{C} 13-\mathrm{O} 2$ & $126.2(4)$ \\
\hline $\mathrm{C} 2-\mathrm{C} 1-\mathrm{Ru} 1$ & $71.2(2)$ & $\mathrm{O} 1-\mathrm{C} 13-\mathrm{C} 11$ & $119.6(3)$ \\
\hline $\mathrm{C} 6-\mathrm{C} 1-\mathrm{Ru} 1$ & $71.0(2)$ & $\mathrm{O} 2-\mathrm{C} 13-\mathrm{C} 11$ & $114.2(3)$ \\
\hline $\mathrm{C} 7-\mathrm{C} 1-\mathrm{Ru} 1$ & $128.0(3)$ & $\mathrm{C} 13-\mathrm{O} 2-\mathrm{Ru} 1$ & $117.7(2)$ \\
\hline $\mathrm{C} 1-\mathrm{C} 2-\mathrm{C} 3$ & $120.6(4)$ & $\mathrm{C} 15-\mathrm{C} 14-\mathrm{O} 3$ & $106.4(6)$ \\
\hline $\mathrm{C} 1-\mathrm{C} 2-\mathrm{Ru} 1$ & $71.4(2)$ & $\mathrm{C} 15-\mathrm{C} 14-\mathrm{C} 16$ & $110.8(7)$ \\
\hline $\mathrm{C} 3-\mathrm{C} 2-\mathrm{Ru} 1$ & $70.6(2)$ & $\mathrm{O} 3-\mathrm{C} 14-\mathrm{C} 16$ & $110.4(5)$ \\
\hline $\mathrm{C} 1-\mathrm{C} 2-\mathrm{H} 2$ & 119.7 & $\mathrm{C} 15-\mathrm{C} 14-\mathrm{C} 17$ & $118.3(9)$ \\
\hline
\end{tabular}




\begin{tabular}{|c|c|c|c|}
\hline $\mathrm{C} 3-\mathrm{C} 2-\mathrm{H} 2$ & 119.7 & $\mathrm{O} 3-\mathrm{C} 14-\mathrm{C} 17$ & $104.3(5)$ \\
\hline $\mathrm{Ru} 1-\mathrm{C} 2-\mathrm{H} 2$ & 131.2 & $\mathrm{C} 16-\mathrm{C} 14-\mathrm{C} 17$ & $106.4(6)$ \\
\hline $\mathrm{C} 4-\mathrm{C} 3-\mathrm{C} 2$ & $121.1(4)$ & $\mathrm{C} 14-\mathrm{C} 16-\mathrm{H} 16 \mathrm{~A}$ & 109.5 \\
\hline $\mathrm{C} 4-\mathrm{C} 3-\mathrm{Ru} 1$ & $72.1(2)$ & $\mathrm{C} 14-\mathrm{C} 16-\mathrm{H} 16 \mathrm{~B}$ & 109.5 \\
\hline $\mathrm{C} 2-\mathrm{C} 3-\mathrm{Ru} 1$ & $71.2(2)$ & $\mathrm{H} 16 \mathrm{~A}-\mathrm{C} 16-\mathrm{H} 16 \mathrm{~B}$ & 109.5 \\
\hline $\mathrm{C} 4-\mathrm{C} 3-\mathrm{H} 3 \mathrm{~A}$ & 119.5 & $\mathrm{C} 14-\mathrm{C} 16-\mathrm{H} 16 \mathrm{C}$ & 109.5 \\
\hline $\mathrm{C} 2-\mathrm{C} 3-\mathrm{H} 3 \mathrm{~A}$ & 119.5 & $\mathrm{H} 16 \mathrm{~A}-\mathrm{C} 16-\mathrm{H} 16 \mathrm{C}$ & 109.5 \\
\hline $\mathrm{Ru} 1-\mathrm{C} 3-\mathrm{H} 3 \mathrm{~A}$ & 129.9 & $\mathrm{H} 16 \mathrm{~B}-\mathrm{C} 16-\mathrm{H} 16 \mathrm{C}$ & 109.5 \\
\hline $\mathrm{C} 3-\mathrm{C} 4-\mathrm{C} 5$ & $117.7(4)$ & $\mathrm{C} 14-\mathrm{C} 17-\mathrm{H} 17 \mathrm{~A}$ & 109.5 \\
\hline $\mathrm{C} 3-\mathrm{C} 4-\mathrm{C} 10$ & $121.1(4)$ & $\mathrm{C} 14-\mathrm{C} 17-\mathrm{H} 17 \mathrm{~B}$ & 109.5 \\
\hline $\mathrm{C} 5-\mathrm{C} 4-\mathrm{C} 10$ & $121.2(4)$ & $\mathrm{H} 17 \mathrm{~A}-\mathrm{C} 17-\mathrm{H} 17 \mathrm{~B}$ & 109.5 \\
\hline $\mathrm{C} 3-\mathrm{C} 4-\mathrm{Ru} 1$ & $70.2(2)$ & $\mathrm{C} 14-\mathrm{C} 17-\mathrm{H} 17 \mathrm{C}$ & 109.5 \\
\hline $\mathrm{C} 5-\mathrm{C} 4-\mathrm{Ru} 1$ & $71.0(2)$ & $\mathrm{H} 17 \mathrm{~A}-\mathrm{C} 17-\mathrm{H} 17 \mathrm{C}$ & 109.5 \\
\hline $\mathrm{C} 10-\mathrm{C} 4-\mathrm{Ru} 1$ & $129.7(3)$ & $\mathrm{H} 17 \mathrm{~B}-\mathrm{C} 17-\mathrm{H} 17 \mathrm{C}$ & 109.5 \\
\hline $\mathrm{C} 6-\mathrm{C} 5-\mathrm{C} 4$ & $121.3(4)$ & $\mathrm{C} 14-\mathrm{C} 15-\mathrm{H} 15 \mathrm{~A}$ & 109.5 \\
\hline $\mathrm{C} 6-\mathrm{C} 5-\mathrm{Ru} 1$ & $71.2(2)$ & $\mathrm{C} 14-\mathrm{C} 15-\mathrm{H} 15 \mathrm{~B}$ & 109.5 \\
\hline $\mathrm{C} 4-\mathrm{C} 5-\mathrm{Ru} 1$ & $71.1(2)$ & $\mathrm{H} 15 \mathrm{~A}-\mathrm{C} 15-\mathrm{H} 15 \mathrm{~B}$ & 109.5 \\
\hline $\mathrm{C} 6-\mathrm{C} 5-\mathrm{H} 5$ & 119.4 & $\mathrm{C} 14-\mathrm{C} 15-\mathrm{H} 15 \mathrm{C}$ & 109.5 \\
\hline $\mathrm{C} 4-\mathrm{C} 5-\mathrm{H} 5$ & 119.4 & $\mathrm{H} 15 \mathrm{~A}-\mathrm{C} 15-\mathrm{H} 15 \mathrm{C}$ & 109.5 \\
\hline $\mathrm{Ru} 1-\mathrm{C} 5-\mathrm{H} 5$ & 131.3 & $\mathrm{H} 15 \mathrm{~B}-\mathrm{C} 15-\mathrm{H} 15 \mathrm{C}$ & 109.5 \\
\hline $\mathrm{C} 5-\mathrm{C} 6-\mathrm{C} 1$ & $121.1(4)$ & $\mathrm{C} 14-\mathrm{O} 3-\mathrm{H} 3$ & 109.5 \\
\hline $\mathrm{N} 1-\mathrm{Ru} 1-\mathrm{C} 1-\mathrm{C} 2$ & $57.5(3)$ & $\mathrm{C} 11-\mathrm{Ru} 1-\mathrm{C} 4-\mathrm{C} 10$ & $-26.2(4)$ \\
\hline $\mathrm{O} 2-\mathrm{Ru} 1-\mathrm{C} 1-\mathrm{C} 2$ & $133.3(2)$ & $\mathrm{C} 3-\mathrm{C} 4-\mathrm{C} 5-\mathrm{C} 6$ & $-1.5(6)$ \\
\hline $\mathrm{C} 3-\mathrm{Ru} 1-\mathrm{C} 1-\mathrm{C} 2$ & $-28.8(2)$ & $\mathrm{C} 10-\mathrm{C} 4-\mathrm{C} 5-\mathrm{C} 6$ & $178.2(4)$ \\
\hline $\mathrm{C} 6-\mathrm{Ru} 1-\mathrm{C} 1-\mathrm{C} 2$ & $-130.2(4)$ & $\mathrm{Ru} 1-\mathrm{C} 4-\mathrm{C} 5-\mathrm{C} 6$ & $52.6(4)$ \\
\hline $\mathrm{C} 5-\mathrm{Ru} 1-\mathrm{C} 1-\mathrm{C} 2$ & $-102.3(3)$ & $\mathrm{C} 3-\mathrm{C} 4-\mathrm{C} 5-\mathrm{Ru} 1$ & $-54.1(3)$ \\
\hline $\mathrm{C} 4-\mathrm{Ru} 1-\mathrm{C} 1-\mathrm{C} 2$ & $-65.6(3)$ & $\mathrm{C} 10-\mathrm{C} 4-\mathrm{C} 5-\mathrm{Ru} 1$ & $125.5(4)$ \\
\hline $\mathrm{C} 11-\mathrm{Ru} 1-\mathrm{C} 1-\mathrm{C} 2$ & $-145.2(2)$ & $\mathrm{N} 1-\mathrm{Ru} 1-\mathrm{C} 5-\mathrm{C} 6$ & $-147.6(4)$ \\
\hline $\mathrm{N} 1-\mathrm{Ru} 1-\mathrm{C} 1-\mathrm{C} 6$ & $-172.3(2)$ & $\mathrm{O} 2-\mathrm{Ru} 1-\mathrm{C} 5-\mathrm{C} 6$ & $47.9(3)$ \\
\hline $\mathrm{O} 2-\mathrm{Ru} 1-\mathrm{C} 1-\mathrm{C} 6$ & $-96.5(2)$ & $\mathrm{C} 3-\mathrm{Ru} 1-\mathrm{C} 5-\mathrm{C} 6$ & $-103.8(3)$ \\
\hline $\mathrm{C} 3-\mathrm{Ru} 1-\mathrm{C} 1-\mathrm{C} 6$ & $101.4(3)$ & $\mathrm{C} 2-\mathrm{Ru} 1-\mathrm{C} 5-\mathrm{C} 6$ & $-66.0(3)$ \\
\hline $\mathrm{C} 2-\mathrm{Ru} 1-\mathrm{C} 1-\mathrm{C} 6$ & $130.2(4)$ & $\mathrm{C} 1-\mathrm{Ru} 1-\mathrm{C} 5-\mathrm{C} 6$ & $-28.8(2)$ \\
\hline $\mathrm{C} 5-\mathrm{Ru} 1-\mathrm{C} 1-\mathrm{C} 6$ & $27.9(2)$ & $\mathrm{C} 4-\mathrm{Ru} 1-\mathrm{C} 5-\mathrm{C} 6$ & $-134.2(4)$ \\
\hline $\mathrm{C} 4-\mathrm{Ru} 1-\mathrm{C} 1-\mathrm{C} 6$ & $64.7(3)$ & $\mathrm{C} 11-\mathrm{Ru} 1-\mathrm{C} 5-\mathrm{C} 6$ & $133.5(2)$ \\
\hline $\mathrm{C} 11-\mathrm{Ru} 1-\mathrm{C} 1-\mathrm{C} 6$ & $-14.9(4)$ & $\mathrm{N} 1-\mathrm{Ru} 1-\mathrm{C} 5-\mathrm{C} 4$ & $-13.4(6)$ \\
\hline $\mathrm{N} 1-\mathrm{Ru} 1-\mathrm{C} 1-\mathrm{C} 7$ & $-60.3(4)$ & $\mathrm{O} 2-\mathrm{Ru} 1-\mathrm{C} 5-\mathrm{C} 4$ & $-178.0(2)$ \\
\hline $\mathrm{O} 2-\mathrm{Ru} 1-\mathrm{C} 1-\mathrm{C} 7$ & $15.5(4)$ & $\mathrm{C} 3-\mathrm{Ru} 1-\mathrm{C} 5-\mathrm{C} 4$ & $30.3(3)$ \\
\hline $\mathrm{C} 3-\mathrm{Ru} 1-\mathrm{C} 1-\mathrm{C} 7$ & $-146.6(4)$ & $\mathrm{C} 2-\mathrm{Ru} 1-\mathrm{C} 5-\mathrm{C} 4$ & $68.2(3)$ \\
\hline $\mathrm{C} 2-\mathrm{Ru} 1-\mathrm{C} 1-\mathrm{C} 7$ & $-117.8(5)$ & $\mathrm{C} 1-\mathrm{Ru} 1-\mathrm{C} 5-\mathrm{C} 4$ & $105.3(3)$ \\
\hline $\mathrm{C} 6-\mathrm{Ru} 1-\mathrm{C} 1-\mathrm{C} 7$ & $112.0(5)$ & $\mathrm{C} 6-\mathrm{Ru} 1-\mathrm{C} 5-\mathrm{C} 4$ & $134.2(4)$ \\
\hline $\mathrm{C} 5-\mathrm{Ru} 1-\mathrm{C} 1-\mathrm{C} 7$ & $139.9(4)$ & $\mathrm{Cl1}-\mathrm{Ru} 1-\mathrm{C} 5-\mathrm{C} 4$ & $-92.4(2)$ \\
\hline $\mathrm{C} 4-\mathrm{Ru} 1-\mathrm{C} 1-\mathrm{C} 7$ & $176.6(4)$ & $\mathrm{C} 4-\mathrm{C} 5-\mathrm{C} 6-\mathrm{C} 1$ & $0.3(6)$ \\
\hline $\mathrm{C} 11-\mathrm{Ru} 1-\mathrm{C} 1-\mathrm{C} 7$ & $97.1(4)$ & $\mathrm{Ru} 1-\mathrm{C} 5-\mathrm{C} 6-\mathrm{C} 1$ & $52.9(3)$ \\
\hline $\mathrm{C} 6-\mathrm{C} 1-\mathrm{C} 2-\mathrm{C} 3$ & $-2.4(6)$ & $\mathrm{C} 4-\mathrm{C} 5-\mathrm{C} 6-\mathrm{Ru} 1$ & $-52.6(3)$ \\
\hline $\mathrm{C} 7-\mathrm{C} 1-\mathrm{C} 2-\mathrm{C} 3$ & $176.3(4)$ & $\mathrm{C} 2-\mathrm{C} 1-\mathrm{C} 6-\mathrm{C} 5$ & $1.7(6)$ \\
\hline $\mathrm{Ru} 1-\mathrm{C} 1-\mathrm{C} 2-\mathrm{C} 3$ & $52.6(3)$ & $\mathrm{C} 7-\mathrm{C} 1-\mathrm{C} 6-\mathrm{C} 5$ & $-177.0(4)$ \\
\hline $\mathrm{C} 6-\mathrm{C} 1-\mathrm{C} 2-\mathrm{Ru} 1$ & $-55.0(3)$ & $\mathrm{Ru} 1-\mathrm{C} 1-\mathrm{C} 6-\mathrm{C} 5$ & $-53.4(3)$ \\
\hline
\end{tabular}




\begin{tabular}{|c|c|}
\hline $\mathrm{C} 7-\mathrm{C} 1-\mathrm{C} 2-\mathrm{Ru} 1$ & $123.6(4)$ \\
\hline $\mathrm{N} 1-\mathrm{Ru} 1-\mathrm{C} 2-\mathrm{C} 1$ & $-132.7(2)$ \\
\hline $\mathrm{O} 2-\mathrm{Ru} 1-\mathrm{C} 2-\mathrm{C} 1$ & $-53.7(3)$ \\
\hline $\mathrm{C} 3-\mathrm{Ru} 1-\mathrm{C} 2-\mathrm{C} 1$ & $133.5(4)$ \\
\hline $\mathrm{C} 6-\mathrm{Ru} 1-\mathrm{C} 2-\mathrm{C} 1$ & $30.7(2)$ \\
\hline $\mathrm{C} 5-\mathrm{Ru} 1-\mathrm{C} 2-\mathrm{C} 1$ & $67.2(3)$ \\
\hline $\mathrm{C} 4-\mathrm{Ru} 1-\mathrm{C} 2-\mathrm{C} 1$ & $104.8(3)$ \\
\hline $\mathrm{C} 11-\mathrm{Ru} 1-\mathrm{C} 2-\mathrm{C} 1$ & $134.7(3)$ \\
\hline $\mathrm{N} 1-\mathrm{Ru} 1-\mathrm{C} 2-\mathrm{C} 3$ & $93.8(2)$ \\
\hline $\mathrm{O} 2-\mathrm{Ru} 1-\mathrm{C} 2-\mathrm{C} 3$ & $172.8(2)$ \\
\hline $\mathrm{C} 1-\mathrm{Ru} 1-\mathrm{C} 2-\mathrm{C} 3$ & $-133.5(4)$ \\
\hline $\mathrm{C} 6-\mathrm{Ru} 1-\mathrm{C} 2-\mathrm{C} 3$ & $-102.8(3)$ \\
\hline $\mathrm{C} 5-\mathrm{Ru} 1-\mathrm{C} 2-\mathrm{C} 3$ & $-66.3(3)$ \\
\hline $\mathrm{C} 4-\mathrm{Ru} 1-\mathrm{C} 2-\mathrm{C} 3$ & $-28.7(2)$ \\
\hline $\mathrm{C} 11-\mathrm{Ru} 1-\mathrm{C} 2-\mathrm{C} 3$ & $1.2(5)$ \\
\hline $\mathrm{C} 1-\mathrm{C} 2-\mathrm{C} 3-\mathrm{C} 4$ & $1.2(6)$ \\
\hline $\mathrm{Ru} 1-\mathrm{C} 2-\mathrm{C} 3-\mathrm{C} 4$ & $54.2(3)$ \\
\hline $\mathrm{C} 1-\mathrm{C} 2-\mathrm{C} 3-\mathrm{Ru} 1$ & $-53.0(3)$ \\
\hline $\mathrm{N} 1-\mathrm{Ru} 1-\mathrm{C} 3-\mathrm{C} 4$ & $135.8(3)$ \\
\hline $\mathrm{O} 2-\mathrm{Ru} 1-\mathrm{C} 3-\mathrm{C} 4$ & $-147.6(3)$ \\
\hline $\mathrm{C} 2-\mathrm{Ru} 1-\mathrm{C} 3-\mathrm{C} 4$ & $-133.1(4)$ \\
\hline $\mathrm{C} 1-\mathrm{Ru} 1-\mathrm{C} 3-\mathrm{C} 4$ & $-104.8(3)$ \\
\hline $\mathrm{C} 6-\mathrm{Ru} 1-\mathrm{C} 3-\mathrm{C} 4$ & $-66.8(3)$ \\
\hline $\mathrm{C} 5-\mathrm{Ru} 1-\mathrm{C} 3-\mathrm{C} 4$ & $-30.4(3)$ \\
\hline $\mathrm{C} 11-\mathrm{Ru} 1-\mathrm{C} 3-\mathrm{C} 4$ & $47.4(3)$ \\
\hline $\mathrm{N} 1-\mathrm{Ru} 1-\mathrm{C} 3-\mathrm{C} 2$ & $-91.1(2)$ \\
\hline $\mathrm{O} 2-\mathrm{Ru} 1-\mathrm{C} 3-\mathrm{C} 2$ & $-14.5(4)$ \\
\hline $\mathrm{C} 1-\mathrm{Ru} 1-\mathrm{C} 3-\mathrm{C} 2$ & $28.3(2)$ \\
\hline $\mathrm{C} 6-\mathrm{Ru} 1-\mathrm{C} 3-\mathrm{C} 2$ & $66.3(3)$ \\
\hline $\mathrm{C} 5-\mathrm{Ru} 1-\mathrm{C} 3-\mathrm{C} 2$ & $102.7(3)$ \\
\hline $\mathrm{C} 4-\mathrm{Ru} 1-\mathrm{C} 3-\mathrm{C} 2$ & $133.1(4)$ \\
\hline $\mathrm{C} 11-\mathrm{Ru} 1-\mathrm{C} 3-\mathrm{C} 2$ & $-179.5(2)$ \\
\hline $\mathrm{C} 2-\mathrm{C} 3-\mathrm{C} 4-\mathrm{C} 5$ & $0.8(6)$ \\
\hline $\mathrm{Ru} 1-\mathrm{C} 3-\mathrm{C} 4-\mathrm{C} 5$ & $54.6(3)$ \\
\hline $\mathrm{C} 2-\mathrm{C} 3-\mathrm{C} 4-\mathrm{C} 10$ & $-178.9(4)$ \\
\hline $\mathrm{Ru} 1-\mathrm{C} 3-\mathrm{C} 4-\mathrm{C} 10$ & $-125.1(4)$ \\
\hline $\mathrm{C} 2-\mathrm{C} 3-\mathrm{C} 4-\mathrm{Ru} 1$ & $-53.8(3)$ \\
\hline $\mathrm{N} 1-\mathrm{Ru} 1-\mathrm{C} 4-\mathrm{C} 3$ & $-55.1(3)$ \\
\hline $\mathrm{O} 2-\mathrm{Ru} 1-\mathrm{C} 4-\mathrm{C} 3$ & $135.3(4)$ \\
\hline $\mathrm{C} 2-\mathrm{Ru} 1-\mathrm{C} 4-\mathrm{C} 3$ & $29.0(3)$ \\
\hline $\mathrm{C} 1-\mathrm{Ru} 1-\mathrm{C} 4-\mathrm{C} 3$ & $65.4(3)$ \\
\hline $\mathrm{C} 6-\mathrm{Ru} 1-\mathrm{C} 4-\mathrm{C} 3$ & $102.6(3)$ \\
\hline $\mathrm{C} 5-\mathrm{Ru} 1-\mathrm{C} 4-\mathrm{C} 3$ & $130.3(4)$ \\
\hline $\mathrm{C} 11-\mathrm{Ru} 1-\mathrm{C} 4-\mathrm{C} 3$ & $-140.7(2)$ \\
\hline $\mathrm{N} 1-\mathrm{Ru} 1-\mathrm{C} 4-\mathrm{C} 5$ & $174.6(2)$ \\
\hline $\mathrm{O} 2-\mathrm{Ru} 1-\mathrm{C} 4-\mathrm{C} 5$ & $5.0(6)$ \\
\hline $\mathrm{C} 3-\mathrm{Ru} 1-\mathrm{C} 4-\mathrm{C} 5$ & $-130.3(4)$ \\
\hline $\mathrm{C} 2-\mathrm{Ru} 1-\mathrm{C} 4-\mathrm{C} 5$ & $-101.3(3)$ \\
\hline
\end{tabular}

\begin{tabular}{|c|c|}
\hline $\mathrm{C} 2-\mathrm{C} 1-\mathrm{C} 6-\mathrm{Ru} 1$ & $55.1(3)$ \\
\hline $\mathrm{C} 7-\mathrm{C} 1-\mathrm{C} 6-\mathrm{Ru} 1$ & $-123.6(4)$ \\
\hline $\mathrm{N} 1-\mathrm{Ru} 1-\mathrm{C} 6-\mathrm{C} 5$ & $151.4(3)$ \\
\hline $\mathrm{O} 2-\mathrm{Ru} 1-\mathrm{C} 6-\mathrm{C} 5$ & $-140.9(2)$ \\
\hline $\mathrm{C} 3-\mathrm{Ru} 1-\mathrm{C} 6-\mathrm{C} 5$ & $65.7(3)$ \\
\hline $\mathrm{C} 2-\mathrm{Ru} 1-\mathrm{C} 6-\mathrm{C} 5$ & $103.5(3)$ \\
\hline $\mathrm{C} 1-\mathrm{Ru} 1-\mathrm{C} 6-\mathrm{C} 5$ & $133.7(4)$ \\
\hline $\mathrm{C} 4-\mathrm{Ru} 1-\mathrm{C} 6-\mathrm{C} 5$ & $28.3(2)$ \\
\hline $\mathrm{C} 11-\mathrm{Ru} 1-\mathrm{C} 6-\mathrm{C} 5$ & $-53.7(3)$ \\
\hline $\mathrm{N} 1-\mathrm{Ru} 1-\mathrm{C} 6-\mathrm{C} 1$ & $17.7(5)$ \\
\hline $\mathrm{O} 2-\mathrm{Ru} 1-\mathrm{C} 6-\mathrm{C} 1$ & $85.4(2)$ \\
\hline $\mathrm{C} 3-\mathrm{Ru} 1-\mathrm{C} 6-\mathrm{C} 1$ & $-68.0(2)$ \\
\hline $\mathrm{C} 2-\mathrm{Ru} 1-\mathrm{C} 6-\mathrm{C} 1$ & $-30.1(2)$ \\
\hline $\mathrm{C} 5-\mathrm{Ru} 1-\mathrm{C} 6-\mathrm{C} 1$ & $-133.7(4)$ \\
\hline $\mathrm{C} 4-\mathrm{Ru} 1-\mathrm{C} 6-\mathrm{C} 1$ & $-105.3(3)$ \\
\hline $\mathrm{C} 11-\mathrm{Ru} 1-\mathrm{C} 6-\mathrm{C} 1$ & $172.66(19)$ \\
\hline $\mathrm{C} 2-\mathrm{C} 1-\mathrm{C} 7-\mathrm{C} 8$ & $97.1(5)$ \\
\hline $\mathrm{C} 6-\mathrm{C} 1-\mathrm{C} 7-\mathrm{C} 8$ & $-84.3(5)$ \\
\hline $\mathrm{Ru} 1-\mathrm{C} 1-\mathrm{C} 7-\mathrm{C} 8$ & $-171.7(3)$ \\
\hline $\mathrm{C} 2-\mathrm{C} 1-\mathrm{C} 7-\mathrm{C} 9$ & $-26.2(6)$ \\
\hline $\mathrm{C} 6-\mathrm{C} 1-\mathrm{C} 7-\mathrm{C} 9$ & $152.5(4)$ \\
\hline $\mathrm{Ru} 1-\mathrm{C} 1-\mathrm{C} 7-\mathrm{C} 9$ & $65.0(5)$ \\
\hline $\mathrm{O} 2-\mathrm{Ru} 1-\mathrm{N} 1-\mathrm{C} 12$ & $-177.7(4)$ \\
\hline $\mathrm{C} 3-\mathrm{Ru} 1-\mathrm{N} 1-\mathrm{C} 12$ & $-24.5(4)$ \\
\hline $\mathrm{C} 2-\mathrm{Ru} 1-\mathrm{N} 1-\mathrm{C} 12$ & $-63.0(3)$ \\
\hline $\mathrm{C} 1-\mathrm{Ru} 1-\mathrm{N} 1-\mathrm{C} 12$ & $-94.1(4)$ \\
\hline $\mathrm{C} 6-\mathrm{Ru} 1-\mathrm{N} 1-\mathrm{C} 12$ & $-106.7(5)$ \\
\hline $\mathrm{C} 5-\mathrm{Ru} 1-\mathrm{N} 1-\mathrm{C} 12$ & $15.7(6)$ \\
\hline $\mathrm{C} 4-\mathrm{Ru} 1-\mathrm{N} 1-\mathrm{C} 12$ & $6.0(4)$ \\
\hline $\mathrm{Cl1}-\mathrm{Ru} 1-\mathrm{N} 1-\mathrm{C} 12$ & $95.8(3)$ \\
\hline $\mathrm{O} 2-\mathrm{Ru} 1-\mathrm{N} 1-\mathrm{C} 11$ & $4.7(3)$ \\
\hline $\mathrm{C} 3-\mathrm{Ru} 1-\mathrm{N} 1-\mathrm{C} 11$ & $157.9(3)$ \\
\hline $\mathrm{C} 2-\mathrm{Ru} 1-\mathrm{N} 1-\mathrm{C} 11$ & $119.3(3)$ \\
\hline $\mathrm{C} 1-\mathrm{Ru} 1-\mathrm{N} 1-\mathrm{C} 11$ & $88.2(3)$ \\
\hline $\mathrm{C} 6-\mathrm{Ru} 1-\mathrm{N} 1-\mathrm{C} 11$ & $75.6(5)$ \\
\hline $\mathrm{C} 5-\mathrm{Ru} 1-\mathrm{N} 1-\mathrm{C} 11$ & $-161.9(4)$ \\
\hline $\mathrm{C} 4-\mathrm{Ru} 1-\mathrm{N} 1-\mathrm{C} 11$ & $-171.7(3)$ \\
\hline $\mathrm{Cl} 1-\mathrm{Ru} 1-\mathrm{N} 1-\mathrm{C} 11$ & $-81.8(3)$ \\
\hline $\mathrm{C} 12-\mathrm{N} 1-\mathrm{C} 11-\mathrm{C} 12^{\mathrm{i}}$ & $0.3(6)$ \\
\hline $\mathrm{Ru} 1-\mathrm{N} 1-\mathrm{C} 11-\mathrm{C} 12^{\mathrm{i}}$ & $178.2(3)$ \\
\hline $\mathrm{C} 12-\mathrm{N} 1-\mathrm{C} 11-\mathrm{C} 13$ & $-179.6(3)$ \\
\hline $\mathrm{Ru} 1-\mathrm{N} 1-\mathrm{C} 11-\mathrm{C} 13$ & $-1.8(4)$ \\
\hline $\mathrm{C} 11-\mathrm{N} 1-\mathrm{C} 12-\mathrm{C} 11^{\mathrm{i}}$ & $-0.3(6)$ \\
\hline $\mathrm{Ru} 1-\mathrm{N} 1-\mathrm{C} 12-\mathrm{C} 11^{\mathrm{i}}$ & $-177.9(3)$ \\
\hline $\mathrm{N} 1-\mathrm{C} 11-\mathrm{C} 13-\mathrm{O} 1$ & $176.9(4)$ \\
\hline $\mathrm{C} 12^{\mathrm{i}}-\mathrm{C} 11-\mathrm{C} 13-\mathrm{O} 1$ & $-3.0(6)$ \\
\hline $\mathrm{N} 1-\mathrm{C} 11-\mathrm{C} 13-\mathrm{O} 2$ & $-4.7(5)$ \\
\hline $\mathrm{C} 12^{\mathrm{i}}-\mathrm{C} 11-\mathrm{C} 13-\mathrm{O} 2$ & $175.3(4)$ \\
\hline
\end{tabular}




$\begin{array}{llll}\mathrm{C} 1-\mathrm{Ru} 1-\mathrm{C} 4-\mathrm{C} 5 & -64.9(3) & \mathrm{O} 1-\mathrm{C} 13-\mathrm{O} 2-\mathrm{Ru} 1 & -172.8(4) \\ \mathrm{C} 6-\mathrm{Ru} 1-\mathrm{C} 4-\mathrm{C} 5 & -27.7(2) & \mathrm{C} 11-\mathrm{C} 13-\mathrm{O} 2-\mathrm{Ru} 1 & 9.0(4) \\ \mathrm{C} 11-\mathrm{Ru} 1-\mathrm{C} 4-\mathrm{C} 5 & 89.0(2) & \mathrm{N} 1-\mathrm{Ru} 1-\mathrm{O} 2-\mathrm{C} 13 & -7.8(3) \\ \mathrm{N} 1-\mathrm{Ru} 1-\mathrm{C} 4-\mathrm{C} 10 & 59.4(5) & \mathrm{C} 3-\mathrm{Ru} 1-\mathrm{O} 2-\mathrm{C} 13 & -89.2(4) \\ \mathrm{O} 2-\mathrm{Ru} 1-\mathrm{C} 4-\mathrm{C} 10 & -110.3(5) & \mathrm{C} 2-\mathrm{Ru} 1-\mathrm{O} 2-\mathrm{C} 13 & -99.1(3) \\ \mathrm{C} 3-\mathrm{Ru} 1-\mathrm{C} 4-\mathrm{C} 10 & 114.5(5) & \mathrm{C} 1-\mathrm{Ru} 1-\mathrm{O} 2-\mathrm{C} 13 & -128.4(3) \\ \mathrm{C} 2-\mathrm{Ru} 1-\mathrm{C} 4-\mathrm{C} 10 & 143.5(5) & \mathrm{C} 6-\mathrm{Ru} 1-\mathrm{O} 2-\mathrm{C} 13 & -166.6(3) \\ \mathrm{C} 1-\mathrm{Ru} 1-\mathrm{C} 4-\mathrm{C} 10 & 179.9(5) & \mathrm{C} 5-\mathrm{Ru} 1-\mathrm{O} 2-\mathrm{C} 13 & 166.9(3) \\ \mathrm{C} 6-\mathrm{Ru} 1-\mathrm{C} 4-\mathrm{C} 10 & -143.0(5) & \mathrm{C} 4-\mathrm{Ru} 1-\mathrm{O} 2-\mathrm{C} 13 & 163.3(4) \\ \mathrm{C} 5-\mathrm{Ru} 1-\mathrm{C} 4-\mathrm{C} 10 & -115.2(5) & \mathrm{C} 11-\mathrm{Ru} 1-\mathrm{O} 2-\mathrm{C} 13 & 77.9(3)\end{array}$

Symmetry code: (i) $-x,-y,-z$.

Hydrogen-bond geometry $\left(A,{ }^{\circ}\right)$

\begin{tabular}{lllll}
\hline$D-\mathrm{H} \cdots A$ & $D-\mathrm{H}$ & $\mathrm{H} \cdots A$ & $D \cdots A$ & $D-\mathrm{H} \cdots A$ \\
\hline $\mathrm{O} 3-\mathrm{H} 3 \cdots \mathrm{O} 1$ & 0.84 & 1.98 & $2.804(5)$ & 168 \\
\hline
\end{tabular}

